PROCEEDINGS OF THE

AMERICAN MATHEMATICAL SOCIETY

Volume 130, Number 3, Pages 625-630

S 0002-9939(01)06091-9

Article electronically published on June 20, 2001

\title{
A SOLVABLE GROUP WHOSE CHARACTER DEGREE GRAPH HAS DIAMETER 3
}

\author{
MARK L. LEWIS
}

(Communicated by Stephen D. Smith)

\begin{abstract}
We show that there is a solvable group $G$ so that the character degree graph of $G$ has diameter 3 .
\end{abstract}

Given a finite group $G$, the set of character degrees of $G$ is the set $\operatorname{cd}(G)=$ $\{\chi(1) \mid \chi \in \operatorname{Irr}(G)\}$. We define $\rho(G)$ to be the set of all primes that divide degrees in $\operatorname{cd}(G)$. With this set, we associate a graph $\Delta(G)$ called the character degree graph of $G$. The vertex set of $\Delta(G)$ is $\rho(G)$, and there is an edge in $\Delta(G)$ between $p$ and $q$ in $\rho(G)$ if $p q$ divides some degree $a \in \operatorname{cd}(G)$. This graph was first introduced in [8].

In this paper, we are interested in the question of which graphs can occur as $\Delta(G)$ when $G$ is a solvable group. Not all graphs occur in this situation. For example in 10] (or Theorem 18.7 of 9]), Pálfy proved that any set of 3 vertices in $\rho(G)$ spans at least one edge in $\Delta(G)$. This implies that either $\Delta(G)$ has at most two connected components, each of which must be a complete graph, or $\Delta(G)$ has one connected component of diameter at most 3 . In this paper, we will be focusing on the diameter of character degree graphs with one connected component. In [8], they proved that the character degree graphs of solvable graphs have diameter at most 3 .

The best places to find the basic knowledge about these graphs are 2], 3, and 9]. It is mentioned in [3], [8, and [9] that they know of no examples where the diameter is actually 3 . In Theorem 14 (d) of [2], Huppert asked the question: "Is [the diameter of $\Delta(G)$ ] always at most 2?" We are now able to answer Huppert's question negatively. The purpose of this note is to present a solvable group whose degree graph has a diameter 3 .

Theorem. There exists a solvable group $G$ so that the diameter of $\Delta(G)$ is 3 .

We begin by introducing some notation. Let $N$ be a normal subgroup of $G$, and $\mathcal{X}$ be a subset of $\operatorname{Irr}(N)$. We define $\operatorname{Irr}(G \mid \mathcal{X})$ to be the set of characters $\chi \in \operatorname{Irr}(G)$ such that some irreducible constituent of $\chi_{N}$ lies in $\mathcal{X}$. In this spirit, we define $\operatorname{cd}(G \mid \mathcal{X})=\{\chi(1) \mid \chi \in \operatorname{Irr}(G \mid \mathcal{X})\}$. To be consistent with the notation in [6], we set $\operatorname{Irr}(G \mid N)=\operatorname{Irr}\left(G \mid \operatorname{Irr}(N) \backslash\left\{1_{N}\right\}\right)$ and $\operatorname{cd}(G \mid N)=\{\chi(1) \mid \chi \in \operatorname{Irr}(G \mid N)\}$.

We spend the remainder of this paper constructing a group $G$ and showing that it fulfills the requirements of the Theorem. The construction we use to create our group $G$ can be found in Section 4 of $[\underline{5}$. We start by letting $F$ be the field of order

Received by the editors May 19, 2000 and, in revised form, August 23, 2000.

2000 Mathematics Subject Classification. Primary 20C15.

(c)2001 American Mathematical Society 
$2^{15}$, and $F\{X\}$ to be the skew polynomial ring with $X \alpha=\alpha^{2} X$ for every element $\alpha \in F$. We define $R$ to be the ring obtained as the quotient of $F\{X\}$ by the ideal generated by $X^{4}$. Let $x$ be the image in $R$ of $X$. Write $J$ for the Jacobson radical of $R$, and note that $P=1+J$ is a subgroup of the group of units of $R$. Also, the elements of $P$ have the form $1+\alpha_{1} x+\alpha_{2} x^{2}+\alpha_{3} x^{3}$ where the $\alpha_{i} \in F$. The order of $P$ is $2^{45}$.

Let $C$ be the multiplicative group $F^{\times}$. The order of $C$ is $2^{15}-1=7 \cdot 31 \cdot 151$. As in [5], $C$ acts on $P$. It is not difficult to see that this action is

$$
\left(1+\alpha_{1} x+\alpha_{2} x^{2}+\alpha_{3} x^{3}\right) \cdot c=1+\alpha_{1} c x+\alpha_{2} c^{3} x^{2}+\alpha_{3} c^{7} x^{3},
$$

where the $\alpha_{i} \in F, c \in C$, and the multiplication occurs in $F$. Let $T$ be the semidirect product of $C$ acting on $P$. Let $\mathcal{G}$ be the Galois group of $F$ over the prime subfield. Thus, $\mathcal{G}$ is cyclic of order 15 . Relying on [5], for any element $\sigma \in \mathcal{G}$, we have

$$
\left(1+\alpha_{1} x+\alpha_{2} x^{2}+\alpha_{3} x^{3}\right) \cdot \sigma=1+\alpha_{1}{ }^{\sigma} x+\alpha_{2}{ }^{\sigma} x^{2}+\alpha_{3}{ }^{\sigma} x^{3},
$$

where the $\alpha_{i} \in F$, and the action of $\sigma$ on $C$ is just the action of $\sigma$ on $F^{\times}$. It is not difficult to see that $\mathcal{G}$ acts via automorphisms on $T$ where $P$ and $C$ are invariant under this action. Our group $G$ is the semi-direct product of $\mathcal{G}$ acting on $T$.

At this point, we compute the irreducible characters of $G / P \cong C \mathcal{G}$. We know that the subgroup of order 3 in $\mathcal{G}$ centralizes the subgroup of order 31 in $C$ and the subgroup of order 5 in $\mathcal{G}$ centralizes the subgroup of order 7 in $C$. From this, we see that $\operatorname{cd}(G / P)=\{1,3,5,15\}$. (For those who want the complete count, $\operatorname{cd}(G / P)$ contains fifteen linear characters, ten characters of degree 3 , eighteen characters of degree 5 , and 2, 182 characters of degree 15 .)

Following [5], we define $P^{i}=1+J^{i}$. Observe that $P^{1}=P$ and $P^{4}=1$, and $P^{i} / P^{i+1}$ is isomorphic to the additive group of $F$ for $i=1,2,3$. It is easy to see that $P^{i}$ is normal in $G$ for every positive integer $i$. Observe that $C P / P^{3}$ is a Frobenius group with Frobenius kernel $P / P^{3}$, and $P / P^{3}$ is one of the groups studied in 1 and [11. (For the remainder of this paper, we will refer to the work in [11.) Using Theorem 4.4 of [11], we see that $\operatorname{Irr}\left(P / P^{3}\right)$ contains $7 \cdot 31 \cdot 151$ nonprincipal linear characters and $2 \cdot 7 \cdot 31 \cdot 151$ characters of degree $2^{7}$. Since under the action of $C$ these characters lie in orbits of size $7 \cdot 31 \cdot 151$, it follows that $\operatorname{Irr}\left(T / P^{3} \mid P / P^{3}\right)$ contains 1 character of degree $7 \cdot 31 \cdot 151$ and 2 characters of degree $2^{7} \cdot 7 \cdot 31 \cdot 151$. Obviously, these characters are invariant under the action of $\mathcal{G}$, and we obtain 15 characters of degree $7 \cdot 31 \cdot 151$ and 30 characters of degree $2^{7} \cdot 7 \cdot 31 \cdot 151$ in $\operatorname{cd}\left(G / P^{3} \mid P / P^{3}\right)$. At this time, we have shown $\operatorname{cd}\left(G / P^{3}\right)=\left\{1,3,5,3 \cdot 5,7 \cdot 31 \cdot 151,2^{7} \cdot 7 \cdot 31 \cdot 151\right\}$. It is easy to see that $\operatorname{cd}(G)=\operatorname{cd}\left(G / P^{3}\right) \cup \operatorname{cd}\left(G \mid P^{3}\right)$. Since we have computed $\operatorname{cd}\left(G / P^{3}\right)$, to determine the structure of $\Delta(G)$ we must find $\operatorname{cd}\left(G \mid P^{3}\right)$.

Define $B=\left\{1+\beta x^{2} \mid \beta \in F\right\} \subseteq P^{2}$. Observe that $B$ is a $C \mathcal{G}$-invariant subgroup of $P^{2}$ and $P^{2}=B \times P^{3}$. The characters in $\operatorname{Irr}\left(P^{2} \mid P^{3}\right)$ have the form $\nu \times \varphi$ where $\nu \in \operatorname{Irr}(B)$ and $1_{P^{3}} \neq \varphi \in \operatorname{Irr}\left(P^{3}\right)$. To compute $\operatorname{Irr}\left(P \mid P^{3}\right)$, we use the following facts from [5]. If $s=1+\alpha x+i$ and $t=1+\beta x^{2}+j$ where $i \in J^{2}$ and $j \in J^{3}$, then $[s, t]=1+\left(\alpha \beta^{2}-\alpha^{4} \beta\right) x^{3}$ (see Corollary 4.2 of [5]). Because of this fact, we define the map $\langle\cdot, \cdot\rangle: F \times F \rightarrow F$ by $\langle\alpha, \beta\rangle=\alpha \beta^{2}-\alpha^{4} \beta$. It is not difficult to see that $\langle\cdot, \cdot\rangle$ is a bilinear map. In particular, fixing an element $\alpha \in F$, the map $\langle\alpha, \cdot\rangle: F \rightarrow F$ is an additive homomorphism. When $\alpha \neq 0$, the kernel of this homomorphism is $\left\{0, \alpha^{3}\right\}$, and thus, the image $\langle\alpha, F\rangle$ is a hyperplane in $F$. 
If $\alpha^{7}=1$, then $\left(\alpha^{4} \beta\right)^{2}=\alpha \beta^{2}$, and $\langle\alpha, \beta\rangle=\alpha^{4} \beta+\left(\alpha^{4} \beta\right)^{2}$, for every element $\beta \in F$. Observe that the set $[F, \mathcal{G}]=\left\{\gamma+\gamma^{2} \mid \gamma \in F\right\}$ is also a hyperplane of $F$ (in fact, $[F, \mathcal{G}]$ can be seen to be the kernel of the trace map of $F$ ), and when $\alpha^{7}=1$, we deduce that $\langle\alpha, F\rangle=[F, \mathcal{G}]$. For any nonzero element $\alpha \in F$, there exist elements $\alpha_{0}, c \in F$ with $\alpha=\alpha_{0} c$ where $\alpha_{0}{ }^{7}=1$ and $c^{31 \cdot 151}=1$. We can write $\beta=\beta_{0} c^{3}$ for some $\beta_{0} \in F$, then

$$
\langle\alpha, \beta\rangle=\alpha_{0} c\left(\beta_{0} c^{3}\right)^{2}+\left(\alpha_{0} c\right)^{4} \beta_{0} c^{3}=c^{7}\left[\alpha_{0}{ }^{4} \beta_{0}+\left(\alpha_{0}{ }^{4} \beta_{0}\right)^{2}\right] .
$$

We conclude that $\langle\alpha, F\rangle=c^{7}([F, \mathcal{G}])$. Since different values of $c$ give different values of $\alpha$, we see that $F$ contains $31 \cdot 151$ hyperplanes of the form $\langle\alpha, F\rangle$.

Observe that $P^{3}$ is isomorphic to $F$ by $1+\alpha x^{3} \mapsto \alpha$. We will view the kernels of characters in $\operatorname{Irr}\left(P^{3}\right)$ as subspaces of $F$ via this isomorphism. Note for every hyperplane in $F$ there is a unique nonprincipal character whose kernel corresponds to the hyperplane. Any action which permutes these hyperplanes must permute the associated characters in the same fashion. We partition the nonprincipal characters of $\operatorname{Irr}\left(P^{3}\right)$ into two sets. Let $\mathcal{A}$ consist of those characters $\varphi \in \operatorname{Irr}\left(P^{3}\right)$ so that $\operatorname{ker}(\varphi)$ corresponds to $\langle\alpha, F\rangle$ for a nonzero element $\alpha \in F$, and write $\mathcal{B}$ for the remaining nonprincipal characters in $\operatorname{Irr}\left(P^{3}\right)$. Because the set of hyperplanes of the form $\langle\alpha, F\rangle$ is stabilized set-wise by $C \mathcal{G}$, both $\mathcal{A}$ and $\mathcal{B}$ are fixed (set-wise) by $C \mathcal{G}$. In particular, $\mathcal{A}$ is a single orbit of size $31 \cdot 151$ under the action of $C$. Also, $\mathcal{B}$ consists of 6 orbits of size $31 \cdot 151$ under the action of $C$, and $\mathcal{G}$ permutes these 6 orbits like two 3-cycles.

Given a character $\varphi \in \operatorname{Irr}\left(P^{3}\right)$ and an element $\alpha \in F$, we define the character $\varphi_{\alpha} \in \operatorname{Irr}(B)$ by $\varphi_{\alpha}\left(1+\beta x^{2}\right)=\varphi\left(1+\langle\alpha, \beta\rangle x^{3}\right)$. Recall that every character in $P^{2}$ has the form $\mu \times \varphi$ where $\mu \in \operatorname{Irr}(B)$ and $\varphi \in \operatorname{Irr}\left(P^{3}\right)$. Let $s=1+\alpha x+i$ where $i \in J^{2}$, and note that $s^{2} \in P^{2}$. For any element $t=1+\beta x^{2}+\gamma x^{3} \in P^{2}$, we have

$$
\begin{aligned}
(\mu \times \varphi)^{s}(t) & =(\mu \times \varphi)\left(t^{s}\right)=(\mu \times \varphi)\left(1+\beta x^{2}+(\gamma+\langle\alpha, \beta\rangle) x^{3}\right) \\
& =\mu\left(1+\beta x^{2}\right) \varphi\left(1+(\gamma+\langle\alpha, \beta\rangle) x^{3}\right) .
\end{aligned}
$$

On the other hand,

$$
\begin{aligned}
\left(\mu \varphi_{\alpha} \times \varphi\right)(t) & =\mu \varphi_{\alpha}\left(1+\beta x^{2}\right) \varphi\left(1+\gamma x^{3}\right) \\
& =\mu\left(1+\beta x^{2}\right) \varphi\left(1+(\gamma+\langle\alpha, \beta\rangle) x^{3}\right) .
\end{aligned}
$$

This shows $(\mu \times \varphi)^{s}=\mu \varphi_{\alpha} \times \varphi$. Notice that the stabilizer of $\mu \times \varphi$ depends only on $\varphi$. We see that $s$ stabilizes $\mu \times \varphi$ if and only if $\varphi_{\alpha}=1_{B}$. This occurs when the kernel of $\varphi$ has the form $\langle\alpha, F\rangle$, and by definition $\varphi \in \mathcal{A}$.

When $\varphi \in \mathcal{B}$, we showed in the previous paragraph that $P^{2}$ is the stabilizer of $\mu \times \varphi$ for every character $\mu \in \operatorname{Irr}(B)$, and $(\mu \times \varphi)^{P}$ is irreducible. In particular, $\varphi$ is fully ramified with respect to $P / P^{3}$. We deduce that $\operatorname{Irr}(P \mid \mathcal{B})$ consists of $6 \cdot 31 \cdot 151$ characters of degree $2^{15}$. Recall that $C$ partitions $\mathcal{B}$ into 6 orbits of size $31 \cdot 151$. We deduce that $\operatorname{Irr}(T \mid \mathcal{B})$ consists of 42 characters of degree $2^{15} \cdot 31 \cdot 151$. Also, our earlier comments on the action of $\mathcal{G}$ on $\mathcal{B}$ imply that 3 divides the size of every orbit for $\mathcal{G}$ on $\operatorname{Irr}(T \mid \mathcal{B})$. Since the subgroup of order 5 in $\mathcal{G}$ centralizes the subgroup of order 7 in $C$, we conclude that $\operatorname{Irr}(G \mid \mathcal{B})$ consists of 70 characters of degree $2^{15} \cdot 3 \cdot 31 \cdot 151$. In particular, $\operatorname{cd}(G \mid \mathcal{B})=\left\{2^{15} \cdot 3 \cdot 31 \cdot 151\right\}$. 
We saw that $C$ acts transitively on $\mathcal{A}$, so $\operatorname{Irr}(G \mid \mathcal{A})=\operatorname{Irr}(G \mid \varphi)$ where $\varphi$ is the character in $\operatorname{Irr}\left(P^{3}\right)$ whose kernel is $[F, \mathcal{G}]$. To simplify our computations, we partition $\operatorname{Irr}\left(P^{2} \mid \varphi\right)$ into two sets. Write $\mathcal{A}_{1}$ for the $P$-orbit in $\operatorname{Irr}\left(P^{2} \mid \varphi\right)$ that contains $1_{B} \times \varphi$ and $\mathcal{A}_{2}$ for the remaining characters in $\operatorname{Irr}\left(P^{2} \mid \varphi\right)$. It is easy to see that $\operatorname{Irr}(G \mid \mathcal{A})=\operatorname{Irr}(G \mid \varphi)=\operatorname{Irr}\left(G \mid \mathcal{A}_{1}\right) \cup \operatorname{Irr}\left(G \mid \mathcal{A}_{2}\right)$.

Take $S$ to be the stabilizer in $G$ of $1_{B} \times \varphi$. Define $Q$ to be the set of elements $1+\alpha_{1} x+\alpha_{2} x^{2}+\alpha_{3} x^{3}$ where the elements $\alpha_{i}$ all lie in the subfield of order 8 in $F$. Observe that $Q$ is a subgroup of $P$ and $S=Q P^{2}$. Let $C_{7}$ be the subgroup of order 7 in $C$, and observe that $Q$ is invariant under the action of $C_{7}$. Furthermore, $S / P^{2}$ is irreducible under the action of $C_{7}$. Also, the character $1_{B} \times \varphi$ is $C_{7}$-invariant. Using Problem 6.12 of [4, we know that either $1_{B} \times \varphi$ extends to $S$ or $1_{B} \times \varphi$ is fully ramified with respect to $S / P^{2}$. Because $\left|S: P^{2}\right|=8$ is not a square, we conclude that $1_{B} \times \varphi$ extends to $S$. Using Glauberman's lemma, we see that $\varphi$ has a unique $C_{7}$-invariant extension $\hat{\varphi} \in \operatorname{Irr}(S)$. Now, $\hat{\varphi}^{P}$ is a $C_{7}$-invariant irreducible character of $P$ whose degree is $2^{12}$. The remaining 7 characters in $\operatorname{Irr}\left(S \mid 1_{B} \times \varphi\right)$ form an orbit under the action of $C_{7}$. They induce irreducibly to $P$ in a $C_{7}$-orbit of characters whose degrees are $2^{12}$. In $\operatorname{Irr}\left(T \mid \mathcal{A}_{1}\right)$, this yields seven characters of degree $2^{12} \cdot 31 \cdot 151$ and one character of degree $2^{12} \cdot 7 \cdot 31 \cdot 151$. Since $1_{B} \times \varphi$ is $\mathcal{G}$-invariant, the character of degree $2^{12} \cdot 7 \cdot 31 \cdot 151$ extends to 15 characters of this degree in $\operatorname{Irr}(G)$. It is not difficult to see that $\mathcal{G}$ stabilizes one of the characters of degree $2^{12} \cdot 31 \cdot 151$ and permutes the others in two orbits of size 3 . This yields 15 characters in $\operatorname{Irr}(G)$ of degree $2^{12} \cdot 31 \cdot 151$ and 10 characters of degree $2^{12} \cdot 3 \cdot 31 \cdot 151$. We conclude that

$$
\operatorname{cd}\left(G \mid \mathcal{A}_{1}\right)=\left\{2^{12} \cdot 31 \cdot 151,2^{12} \cdot 3 \cdot 31 \cdot 151,2^{12} \cdot 7 \cdot 31 \cdot 151\right\} .
$$

The final case to consider consists of characters in $\mathcal{A}_{2}$. Let $\sigma$ be an element of order 5 in $\mathcal{G}$. It is easy to see that $Q$ is the centralizer in $P$ of $\sigma$. From Fitting's theorem, we have $B=(Q \cap B) \times[B, \sigma]$. Fix $0 \neq \alpha \in F$, and consider an element $\beta$ in the subfield of order 8 in $F$. We will show that $1+\beta x^{2}$ lies in $\operatorname{ker}\left(\varphi_{\alpha}\right)$. When $\beta=0$, this is obviously the case, so we assume that $\beta \neq 0$. This implies $\beta^{7}=1$. Observe that

$$
\langle\alpha, \beta\rangle=\alpha \beta^{2}+\alpha^{4} \beta=\left(\alpha \beta^{2}+\left(\alpha \beta^{2}\right)^{2}\right)+\left(\alpha^{2} \beta^{4}+\left(\alpha^{2} \beta^{4}\right)^{2}\right) \in[F, \mathcal{G}]=\operatorname{ker}(\varphi),
$$

and this proves the claim. We have $2^{12}$ characters of the form $\varphi_{\alpha}$. This implies that $\operatorname{Irr}(B /(Q \cap B))=\left\{\varphi_{\alpha} \mid \alpha \in F\right\}$. Using the earlier direct product, we see that the orbits of $\operatorname{Irr}\left(P^{2} \mid \varphi\right)$ under the action of $P$ are identified with the cosets of $\operatorname{Irr}([B, \sigma])$ as a subgroup of $\operatorname{Irr}(B)$. Since $C_{7}$ acts transitively on the nonidentity elements of $Q \cap B \cong B /[B, \sigma]$, we deduce that $\mathcal{A}_{2}$ is a single orbit under the action of $P C_{7}$, and $\operatorname{Irr}\left(G \mid \mathcal{A}_{2}\right)=\operatorname{Irr}(G \mid \mu \times \varphi)$ where $\mu \times \varphi$ is any character in $\mathcal{A}_{2}$. Fix the character $\mu \in \operatorname{Irr}(B)$ so that $\mu \times \varphi \in \mathcal{A}_{2}$ and the kernel of $\mu$ contains $[B, \sigma]$.

Define $Q^{i}=Q \cap P^{i}$ for $i=1,2,3,4$. Note that $S$ is the stabilizer of $\mu \times \varphi$ in $P$, and $S /\left([B, \sigma] \times P^{3}\right) \cong Q / Q^{3}$. Observe that $Q / Q^{3}$ satisfies the hypotheses of the groups studied in [11. Using those results, we see that $\operatorname{Irr}\left(Q / Q^{3} \mid Q^{2} / Q^{3}\right)$ consists of 14 characters of degree 2. Note that these characters form 2 orbits under the action of $C_{7}$. Thus, $\operatorname{Irr}(S \mid \mu \times \varphi)$ contains 14 characters of degree 2 . These induce irreducibly to 14 characters of degree $2^{13}$ in $\operatorname{Irr}(P)$. We already noted that $C_{7}$ acts faithfully on these characters. It is easy to see that $C_{7}$ in $C$ also acts faithfully on $\operatorname{Irr}(P \mid \mu \times \varphi)$, and $\operatorname{Irr}\left(T \mid \mathcal{A}_{2}\right)$ consists of 2 characters of degree $2^{13} \cdot 7 \cdot 31 \cdot 151$. In 


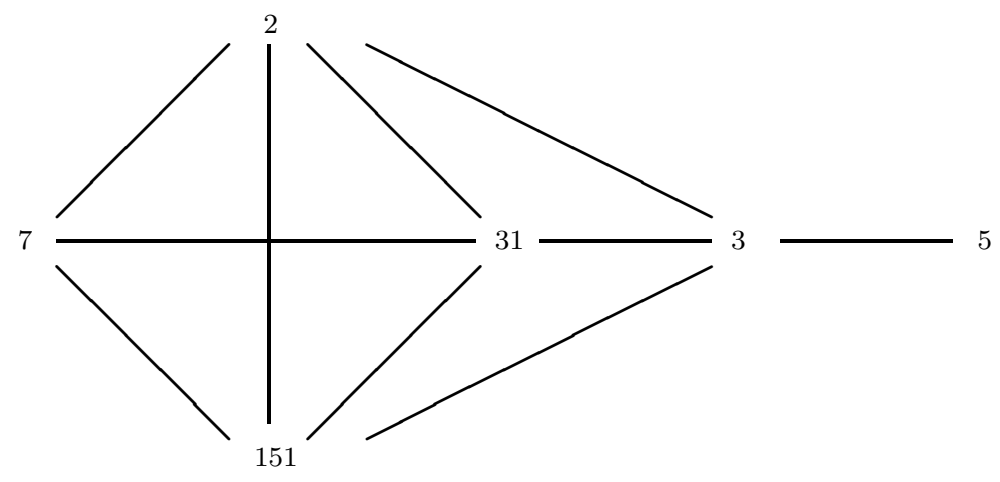

FiguRE 1.

fact, these are the only characters of this degree that lie in $\operatorname{Irr}(T \mid \mathcal{A})$. Therefore, they must be invariant under the action of $\mathcal{G}$, and the only characters $\operatorname{in} \operatorname{Irr}\left(G \mid \mathcal{A}_{2}\right)$ are 30 characters of this degree. In particular, $\operatorname{cd}\left(G \mid \mathcal{A}_{2}\right)=\left\{2^{13} \cdot 7 \cdot 31 \cdot 151\right\}$.

We note that $\operatorname{cd}(G)=\operatorname{cd}\left(G / P^{3}\right) \cup \operatorname{cd}\left(G \mid \mathcal{A}_{1}\right) \cup \operatorname{cd}\left(G \mid \mathcal{A}_{2}\right) \cup \operatorname{cd}(G \mid \mathcal{B})$, and thus, we have shown that

$$
\begin{aligned}
\operatorname{cd}(G)= & \left\{1,3,5,3 \cdot 5,7 \cdot 31 \cdot 151,2^{7} \cdot 7 \cdot 31 \cdot 151,2^{12} \cdot 31 \cdot 151,\right. \\
& \left.2^{12} \cdot 3 \cdot 31 \cdot 151,2^{12} \cdot 7 \cdot 31 \cdot 151,2^{13} \cdot 7 \cdot 31 \cdot 151,2^{15} \cdot 3 \cdot 31 \cdot 151\right\} .
\end{aligned}
$$

Using this set, we determine that $\Delta(G)$ is the graph in Figure 1. It is clear that this graph has diameter 3.

Our group $G$ is quite big. (The order of $G$ is on the order of $10^{19}$.) It is reasonable to ask whether there is a smaller example. Our intuition is that there is no smaller example, but at this time we do not have a proof of this. We do know that $|\rho(G)|$ is as small as possible. When the diameter of $\Delta(G)$ is 3 , it follows that $\rho(G)$ has at least 4 vertices. In the paper [12], Zhang proved that $\Delta(G)$ cannot be a graph with 4 vertices and diameter 3 . In our preprint [7, we show that $\Delta(G)$ cannot be a graph with 5 vertices and diameter 3 . Therefore, in any example, $\rho(G)$ must have size at least 6 , and 6 is the cardinality of $\rho(G)$ in our example.

In conclusion, we would like to consider the possibility of finding other solvable groups whose character degree graphs have diameter 3 . We believe that other such groups can be found among those described in Section 4 of [5]. The construction of these groups is similar to one used to construct $G$ following the Main Theorem. We conjecture that if one uses a field of order $p^{q r}$ where $p$ is a prime power and $q<r$ are distinct primes that do not divide $\left(p^{q r}-1\right) /(p-1)$ and we factor out the ideal generated by $X^{q+1}$, then the resulting groups will have character degree graphs of diameter 3 . Notice that when $p=2$ and $q=3$ a proof similar to the one outlined in this paper should yield a similar result. In fact, our proof can probably be generalized to prove the conjecture for all cases when $q=3$, but difficulties arise in our proof when $q$ is changed from 3 . It seems likely to us that solvable groups whose degree graphs have diameter 3 must be somewhat rare. If this is the case, it should be possible to classify these groups.

I conclude by thanking Don White and John McVey for reading a preprint of this result, and for their helpful comments in writing this article. 


\section{REFERENCES}

[1] A. Hanaki, A condition on lengths of conjugacy classes and character degrees, Osaka J. Math. 33 (1996), 207-216. MR 97b:20007

[2] B. Huppert, Research in representation theory at Mainz (1984-1990), Progr. Math. 95 (1991), 17-36. MR 92c:20011

[3] B. Huppert, Character Theory of Finite Groups, deGruyter Expositions in Mathematics 25, Berlin, 1998. MR 99j:20011

[4] I. M. Isaacs, Character Theory of Finite Groups, Academic Press, New York, 1976. MR 57:417

[5] I. M. Isaacs, Coprime group actions fixing all nonlinear irreducible characters, Can. J. Math. 41 (1989), 68-82. MR 90j:20038

[6] I. M. Isaacs and G. Knutson, Irreducible character degrees and normal subgroups, J. Algebra 199 (1998), 302-326. MR 98m:20013

[7] M. L. Lewis, Solvable groups with degree graphs having 5 vertices and diameter 3, Preprint.

[8] O. Manz, W. Willems, and T. R. Wolf, The diameter of the character degree graph, J. Reine Angew. Math. 402 (1989), 181-198. MR 90i:20007

[9] O. Manz and T. R. Wolf, Representations of Solvable Groups, Cambridge University Press, Cambridge, 1993. MR 95c:20013

[10] P. P. Pálfy, On the character degree graph of solvable groups, I, Period. Math. Hungar. 36 (1998), 61-65. MR 2000c:20019

[11] J. M. Riedl, Character degrees, class sizes, and normal subgroups of a certain class of $p$ groups, J. Algebra 218 (1999), 190-215. MR 2000f:20025

[12] J. Zhang, On a problem by Huppert, Acta Scientiarum Naturalium Universitatis Pekinensis 34 (1998), 143-150. MR 99j:20014

Department of Mathematics and Computer Science, Kent State University, Kent, OHIO 44242

E-mail address: lewis@mcs.kent.edu 